\title{
The concealed morbidity of buried penis: a narrative review of our progress in understanding adult-acquired buried penis as a surgical condition
}

\author{
Christopher J. Staniorski, Paul J. Rusilko^ \\ Department of Urology, University of Pittsburgh Medical Center, Pittsburgh, PA, USA \\ Contributions: (I) Conception and design: All authors; (II) Administrative support: All authors; (III) Provision of study materials or patients: None; \\ (IV) Collection and assembly of data: None; (V) Data analysis and interpretation: None; (VI) Manuscript writing: All authors; (VII) Final approval of \\ manuscript: All authors. \\ Correspondence to: Paul Rusilko, DO, FACS. Department of Urology, University of Pittsburgh Medical Center, 3471 Fifth Ave, Suite 700, Pittsburgh, \\ PA 15213, USA. Email: rusilkopj@upmc.edu.
}

\begin{abstract}
Adult acquired buried penis (AABP) is a condition of entrapment of the phallus resulting most commonly from morbid obesity and formation of cicatrix with other etiologies including genital lymphedema, hidradenitis and trauma. The incidence of this syndrome is invariably connected to the increasing prevalence of obesity. The purpose of this review is to examine the current literature in AABP with a focus on the morbidity of AABP and perioperative management. The discussion and literature surrounding buried penis reconstruction started with the goal of correcting a cosmetic problem and has recently become fairly successful in this aim with an over $85 \%$ rate of successful reconstruction in many series with a more uniform surgical approach. The most recent trends have examined the significant burden of morbidity and even mortality that AABP can place on patients as it contributes to risk of penile cancer, urethral strictures and mood disorders. Studies in this space have shown that surgical repair can be successful in improving quality of life for patients with AABP and the removal of the offending pathophysiology suggests its success in correcting the physical morbidities. New directions for research and management of this condition should include a focus on educating providers and patients to make reconstruction more accessible to patients in need as AABP continues to journey toward mainstream acceptance as a surgical condition.
\end{abstract}

Keywords: Adult acquired buried penis (AABP); reconstruction; penile cancer

Submitted Sep 03, 2020. Accepted for publication Jan 12, 2021.

doi: $10.21037 /$ tau-20-1232

View this article at: http://dx.doi.org/10.21037/tau-20-1232

\section{Introduction}

Adult acquired buried penis (AABP) is the condition of concealment of the male phallus within a suprapubic fat pad or a cicatrix. This most commonly occurs in morbidly obese patients, however significant genital lymphedema, hidradenitis of the genitals and post-circumcision scar tissue can also lead to burying of the phallus. While other conditions have an association with $\mathrm{AABP}$, the trend of increasing obesity in our society, with a prevalence of up to $40.8 \%$ in middle-aged men, brings special concern to this discussion as the suprapubic area, or escutcheon, is a common place for accumulation of adiposity $(1,2)$. The increased size of the escutcheon can lead to an environment of chronic inflammation with infections, difficulties with hygienic voiding and skin breakdown which can further contribute to scar formation (1). These conditions lead

^ ORCID: 0000-0002-5402-6623 

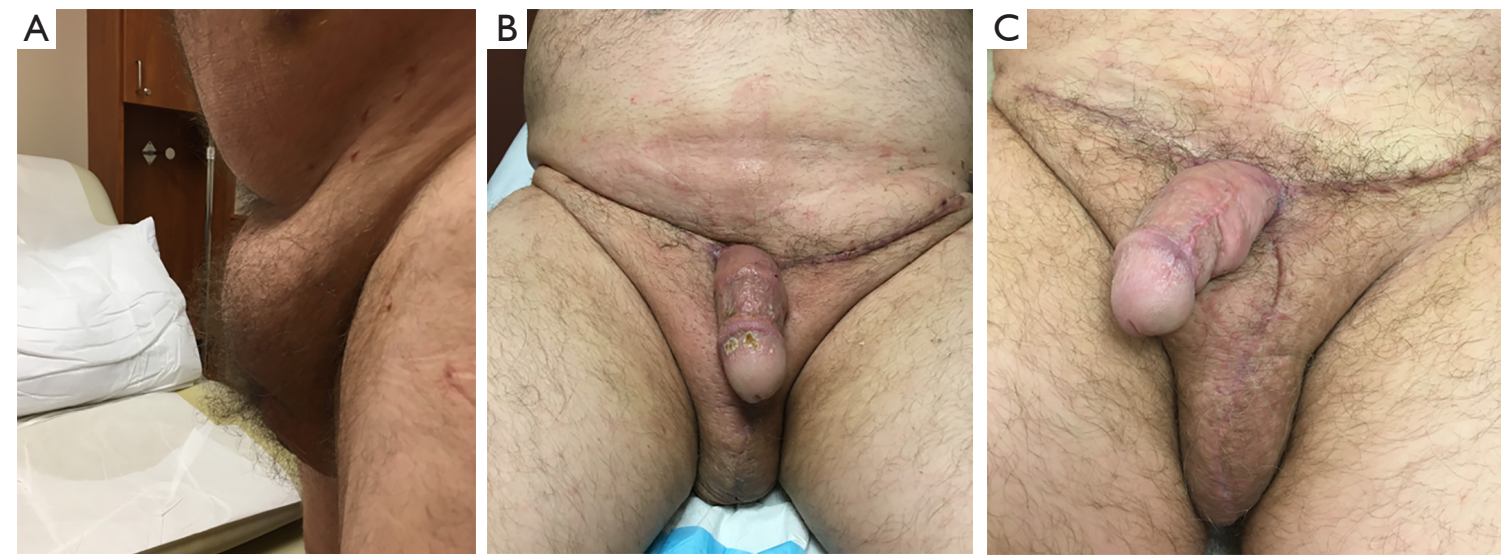

Figure 1 Pre-operative and post-operative AABP repair. (A) Pre-operative image of AABP. (B) Three-month post-operative image from repair including exhuming penis, escutcheonectomy and STSG. (C) One-year post-operative results.

to concerns with sexual function, urinary difficulties, skin infections and irritation and the associated depression and decreased quality of life that drive patients to seek care (3-7). While we can hint at the scope of the problem with the increased incidence of obesity in men and this pathophysiology resulting in a host of medical and psychologic problems, the prevalence of AABP in a review of the literature is not known.

Unlike many other conditions in medicine, the management of buried penis was developed long before we garnered a complete understanding of the scope. The earliest reports of buried penis were in 1917 by Dr. Keyes when he described a condition when the "penis, lacking its proper sheath of skin, lies buried beneath the integument of the abdomen or scrotum". From this report, the primary focus was initially surgical correction with "liberation of the member to avoid urinary infiltration" (8). More modern reports of buried penis were first published in children in the 1970's and transitioning to the adult population largely in the 1990's, with continued focus on surgical correction. The most recent developments in buried penis reconstruction have centered on a somewhat similar approach involving a combination of release of scar tissue and excision of unhealthy penile skin, removal of suprapubic fat pad, or escutcheonectomy, split thickness skin grafting and scrotoplasty (9-13). A standard surgical repair is seen in Figure 1. While this method is fairly standardized among providers, some still attempt to improve with methods such as full thickness grafts and improving perioperative care to transition it to an outpatient setting $(14,15)$. More recently, increasingly sophisticated methods for categorizing AABP have been developed while reconstructive surgeons continue to refine surgical methods.

The most recent trend in the literature is an increasing understanding of the comorbid conditions associated with buried penis ranging from penile cancer and stricture to depression. With these advances, adult acquired buried penis has transitioned from a cosmetic issue to one that requires medical attention due to the host of physical and psychologic effects that can ensue. This review will focus on the most recent literature examining the medical and psychologic comorbidities affecting those with this condition and the challenges that this proposes for providers managing these patients. We present the following article in accordance with the Narrative Review Checklist (available at: http://dx.doi.org/10.21037/tau-20-1232).

\section{Methods}

Relevant articles were identified through PubMed search for keywords including: Adult-acquired buried penis, surgical reconstruction, pre-operative evaluation, quality of life, penile cancer, urethral stricture, depression and surgical outcomes. Review was focused on articles from after 2000 commenting on morbidity of adult acquired buried penis. Given multiple recent reviews of the surgical literature, this review focused on only the most recent surgical techniques following 2015. All articles were published in peer-reviewed journals and were largely retrospective reviews of patient populations or series without randomized controlled trials available in the space. Articles were deemed suitable for inclusion after review by the authors for quality and relevance. This review was limited to articles published in English. 


\section{Understanding the physical morbidity of AABP}

Over recent years, research has uncovered various physical and psychological morbidities associated with AABP. Here, we will review some of the most significant physical effects including increased risk for penile cancer and urethral stricture disease. Interestingly, the most common hypothesized physical morbidities of AABP—skin infection, urinary tract infections and related complications-are not clearly defined in the literature in comparison to the rarer complications of cancer and urethral stricture disease.

Penile cancer is, perhaps, the direst of these morbidities due to the five-year mortality rate of $50 \%$ associated with the condition. AABP creates a host of risk factors for development of penile cancer due to the chronic inflammation made worse by an inability to complete exhume the penis for routine examination. A recent study by Pekala et al. showed an invasive penile cancer prevalence of $7 \%$ in a population of AABP patients with a $35 \%$ prevalence of premalignant lesions in a sizable population of 150 patients (16). Other studies have identified obesity as a risk for penile cancer based on a population based approach in a large cohort (17). In comparison to many other studies in this space, this cohort is fairly large and supported by a population-based study to further emphasize the importance of its consideration. Together, these results encourage physicians to consider this condition in conjunction with buried penis and prompt earlier referral to a center capable of surgical correction and management.

While chronic inflammation can create an environment for development of malignancies, it can also predispose patients to urethral stricture disease and the associated lower urinary tract symptoms, risk for infection and urolithiasis, and obstructive uropathy. Lichen sclerosis associated with $\mathrm{AABP}$ can be a cause of often lengthy urethral strictures most commonly in the anterior urethra, a presentation that is fairly uncommon among the typical patients presenting with stricture disease. Liaw et al. were the first to demonstrate the association of urethral stricture with AABP with $33 \%$ of a population of 39 patients presenting with urethral stricture with the majority (two-thirds) being in the anterior urethra. Of those that underwent treatment for strictures, half underwent dilation and one-quarter had a meatotomy (4). Fuller et al. similarly demonstrated a $31 \%$ prevalence of urethral stricture disease in a population of 42 AABP patients with all strictures in the anterior urethra (6). Interestingly, the average stricture length was $5.8 \mathrm{~cm}$ with over half of these patients having a long segment stricture greater than $6 \mathrm{~cm}$ and requiring Kulkarni urethroplasty prior to formal AABP repair. This requires a significant amount of buccal mucosa for reconstruction and adds to the morbidity and difficulty of reconstruction making this an important consideration. These studies highlight that lichen sclerosis is highly associated with the finding of urethral stricture which supports a causative association between the chronic inflammatory environment of AABP and urethral stricture $(4,6)$. Liaw et al. reported no significant recurrence of stricture disease following initial management.

While these studies highlight the scope of cancer and stricture, they do not comment directly on the benefits of surgical correction. Others have shown significant improvements in hygienic voiding following correction which can break the cycle of chronic inflammation associated with the proposed pathophysiology of these conditions $(3,7,18-20)$. These developments in the understanding of various causes of morbidity and mortality in the AABP population further suggest that this condition is not simply a cosmetic issue and should be considered a risk for significant medical comorbidity.

\section{Sexual and psychologic burden of AABP}

While some studies focus on medical conditions associated with $\mathrm{AABP}$, the quality of life of patients with $\mathrm{AABP}$ is better evaluated in those examining the rates of depression, voiding function and sexual function. In early descriptions of modern buried penis repair, authors commented on the moderate to severe depression, even suicidal ideation, in patients with buried penis (21). Rybak et al. helped to establish the burden of these conditions in the AABP population in a small study of 11 patients showing that pre-operatively, $64 \%$ had clinical depression and $91 \%$ had erectile dysfunction by validated questionnaires (3). Another study evaluating specifically the sexual effects of buried penis demonstrated at least mild impairment in most domains of the international index of erectile function (IIEF) questionnaire pre-operatively (5).

The success of surgery in terms of sexual outcomes has been studied in a number of ways in recent years. Studies have documented improvements in overall function and specifically in domains of arousal/erection and orgasm/ ejaculation $(19,20)$. When using validated questionnaires such as the IIEF, erectile dysfunction was also found to decrease from $91 \%$ to $63 \%$ in one study, while others reported individual improvements in domains of erectile/ orgasmic function, intercourse satisfaction and overall 
satisfaction $(3,5)$. Validated questionnaires from the prostate cancer literature (Expanded Prostate Cancer Index or EPIC) have also been used to assess sexual function with significant improvement in $87.5 \%$ of patients following surgical repair (7). Similarly, urinary function improves after repair. Again using EPIC, $87.5 \%$ of patients were shown to have improved urinary function (7). Others demonstrated that $80-91 \%$ of patients could void standing with adequate stream following repair $(3,18)$. Studies have also shown that patients following repair not only have improvements in subjective voiding difficulty but also in genital hygiene and skin rashes following the procedure $(18,19)$.

These improvements in urinary and sexual function have translated to overall quality of life and depression. Post-bariatric surgery questionnaires have been used to show improvements in embarrassment concerning genital appearance and social issues like difficulties shopping (19). Quality of life was also measured as a single composite score by validated questionnaires and was found to have increased in $91 \%$ of one study's cohort following surgery (3). These data taken together support Rybak et al.'s finding that clinically significant depression decreased from $64 \%$ pre-operatively to $18 \%$ post-operatively in their cohort (3). The simplest parameter of success in these categories is the number who would undergo surgery again and those for whom surgery had a positive impact on their lives. In one published study, $85-92 \%$ of patients would choose to have surgery again and $74-83 \%$ believe that the surgery had a positive impact for them $(18,19)$.

While many of these studies are in small populations of less than twenty patients, the fairly uniform success in these various quality of life domains illustrates the positive impact that buried penis reconstruction can have for patients. Aside from the sample size that afflicts many studies of AABP, the main drawback of these studies are the attempts to apply questionnaires specific to other conditions to AABP. Urinary symptoms of loss of control or irritative symptoms following prostatectomy are much different than the issues of hygienic voiding that apply to AABP. Questions related to quality of stream or ability to expose meatus to void could be more applicable to this population than those related to true stress incontinence. Likewise, some of the questions in the IIEF related to sexual interest, penetration or overall satisfaction may be more related to AABP than those querying the quality and durability of erections. While these studies are adequate for initial analyses, a true validated questionnaire for buried penis quality of life and symptoms could better guide operative decisions and track outcomes as we continue to learn about this condition.

\section{Peri-operative considerations in surgical management of AABP}

While we will discuss the remaining barriers to improving the lives of patients affected by these complications of $\mathrm{AABP}$, the perioperative management is one area that has been refined to improve the delivery of care and reflects our understanding of AABP as a medical as well as cosmetic issue. This includes the development of algorithms for operative management as well as classification of disease severity. Adult acquired buried penis reconstruction can be challenging as each case poses a unique set of circumstances and reconstructive surgeons have a variety of tools available to them. Algorithms for management started with Donatucci et al. who proposed an early intra-operative algorithm for management starting with scar contracture release, proceeding to escutcheonectomy if necessary and continuing with preferential use of adjacent skin for closure of defect (22). Tausch et al. proposed a newer algorithm taking into account the health of the penile skin with groups of viable and non-viable to stratify the need for STSG. A separate group was delineated by genital lymphedema. Each of these was subdivided by whether or not an abdominal component was involved (23). The most recent classification system impacting surgical decision making is shown in Table 1. The Wisconsin Classification system provides a clear progression from intraoperative or preoperative findings connecting these to surgical management (12). With a narrowing consensus on approach to these cases, this simple framework is likely helpful however should not be a substitute for experience in these cases that can often require intraoperative judgement to determine a final plan.

While these algorithms help to guide intraoperative decision making, a recent study published by Pariser et al. expanded the stratification of the complexity of the surgery and linked this to outcomes in their population of 64 patients (Table 2) (13). In their classification, Category III and above were considered complex repairs and made up more than half of the population. Going further, this classification system was associated with outcomes. BMI and high-grade complication rate were significantly higher in high complexity surgeries $(23 \%$ vs. $0 \%$ in category I-II). While no significant differences were established, Category III-V surgeries had a total complication rate of $73 \%$ compared to $50 \%$ in Category I-II. Success rate was $100 \%$ in low complexity reconstruction and $86 \%$ in high 
Table 1 The Wisconsin Classification System of adult acquired buried penis (12)

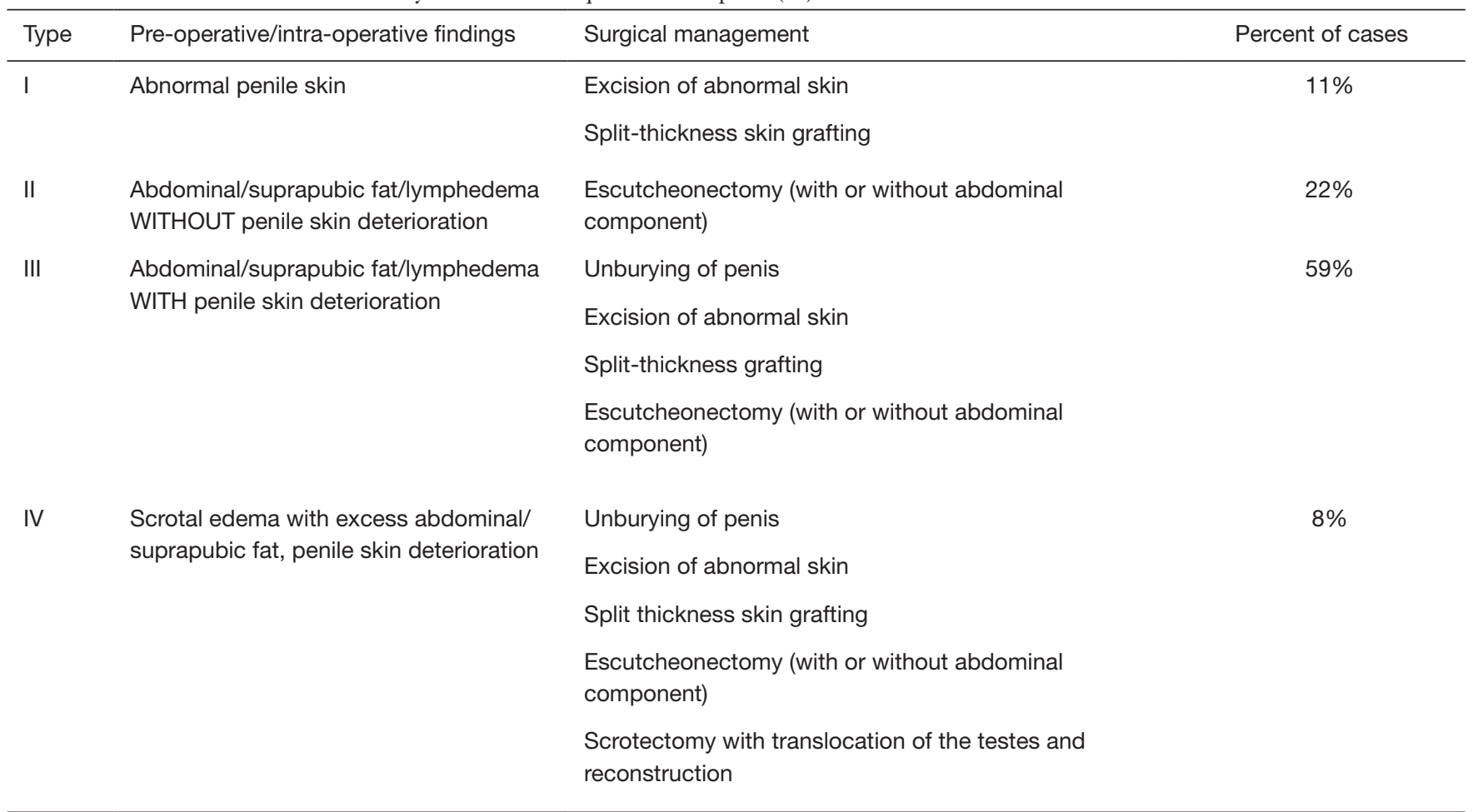

Table 2 Pariser et al. classification of buried penis complexity (13)

\begin{tabular}{|c|c|c|c|c|}
\hline Category & Description & $\begin{array}{l}\text { Prevalence in study } \\
\text { population }\end{array}$ & $\begin{array}{l}\text { Complication rate } \\
\text { (high grade complication) }\end{array}$ & Percent successful \\
\hline I & Penile unburying with local skin flap & $5 \%$ & $50 \%(0 \%)$ & $100 \%$ \\
\hline III & $\begin{array}{l}\text { Scrotal surgery (scrotectomy or } \\
\text { scrotoplasty) }\end{array}$ & $11 \%$ & $73 \%(23 \%)$ & $86 \%$ \\
\hline $\mathrm{V}$ & Abdominal panniculectomy & $6 \%$ & & \\
\hline
\end{tabular}

complexity cases, however this was, again, not a statistically significant finding. The complexity within the high complexity group likely has some variability as those with a panniculectomy are likely more prone to complication than simply an escutcheonectomy. While the small number of patients undergoing these repairs prevents analysis of these subcategories, these considerations are likely something that also weighs on pre-operative counseling. This classification also does not take into account the added complexity of urethral stricture management as detailed above. Correlating this to the Wisconsin Grading system above, Types II-IV would all be considered high complexity reconstructions and be at higher risk for complications. All in all, this classification provides an understanding of the spectrum of buried penis repair that could be used for research, patient counseling and surgical planning.

While complications can be based on the complexity of the surgery, AABP is associated with a number of comorbidities, most notably morbid obesity and diabetes, which can affect outcomes and healing from the procedure. A study by Aubé et al. examined a population of twenty-four patients who underwent $\mathrm{AABP}$ repair with this question 
in mind. They found that abdominal panniculectomy (a high complexity surgery) was associated with Clavien 3 complications with a hazard ratio of 28 . However, they also found that $\mathrm{BMI}>40$ and tobacco smoking were associated with an increased overall complication rate with hazard ratios of 25 and 14.6 respectively (24). As one would think, this suggests that regardless of where patients fall in the above classifications, they bring an independent risk of complications when approaching cases. Hampson et al. also performed analyses in a population of $42 \mathrm{AABP}$ repair patients and determined that complications are associated with higher BMI, actually increasing with every increased point of BMI; prior gastric bypass surgery was also associated with lower rate of complication which is likely a related observation (18).

The results of these recent studies are important for patient management and pre-operative guidance. Success of surgical repair now exceeds $85 \%$ with the refinement of surgical approach and individualized intra-operative approach. However, the procedure has a high complication rate, over $50 \%$. These data can help prepare patients and set expectations for those at higher risk of complication. More importantly, this literature also provides evidence and encouragement that continued efforts for weight loss and tobacco cessation can have dramatic effects in reducing these complications.

\section{Challenges for physicians in managing AABP}

While adult acquired buried penis has beginnings over 100 years ago as a largely cosmetic issue, the research highlighted here has shown the progression of understanding the various physical and emotional issues associated the AABP population as well as an improvement in the way in which reconstructive surgeons' approach and categorize the disease. In many ways, AABP has become a true medical problem associated with the rising rates of obesity and metabolic syndrome, especially in the US. As this disease becomes recognized by more in the medical community, further questions arise regarding the remaining barriers to providing care to patients. Various social, economic and environmental factors can affect a patient's ability to access care for AABP and here, we will highlight these as patient centered and provider centered factors.

For patients, a number of elements could be at play when considering care for AABP, both pre-operatively and post-operatively. Research has shown that depression, embarrassment and self-esteem are all issues that buried penis patients confront when it comes to this condition $(3,20,21)$. Surely, bringing these concerns to providers cannot be an easy conversation for many to have. After presenting to a reconstructive surgeon, other issues could include difficulties in funding the operation through insurance and finding time for the operation.

Other difficulties present post-operatively with the burden of recovery. Typically, AABP reconstruction is an inpatient procedure with stays up to a week or more and variable lengths of bedrest based on individual center practices. Some measures have been taken to improve this as Erpelding et al. evaluated the feasibility of outpatient surgery for AABP in a small population of ten patients. Their population showed universal STSG uptake and similar complication rate between these patients and six patients who spent one night in the hospital (22). A transition to outpatient care could further improve AABP management with decreased healthcare costs and hospital exposures for patients on admission, however larger studies should be conducted to fully examine this practice. While the goal of standardization of outpatient surgery may be difficult in a population with significant comorbidities associated with obesity who undergo a range of reconstructions, this study should encourage shorter hospital stays in this population to further lessen the burden on patients during recovery (15). Wound care following surgery can further tax patient resources and families contributing to the barriers in management.

Some barriers to care are also present for providers. As this condition is only now being truly uncovered, many could have difficulty in finding direction. In a search of reference material such as UpToDate, American Urologic Association guidelines or statements and the pre-eminent text in Urology (Campbell Walsh Wein Urology, 12th Edition), it is difficult to find guidance for management of AABP. This stems from the small number of repairs that are done which can be seen as a limitation throughout the studies highlighted in this manuscript. Large cohorts are those with greater than fifty patients and most research is retrospective. This makes studying the data for trends difficult and makes us rely heavily on expert opinion and experience.

Given this environment, the natural reflex for primary care providers would be to suggest weight loss and treatment of metabolic syndrome through counseling on diet and exercise. This is a heroic task for any provider in a short session in clinic. The largest studies of attempts at weight loss come from the diabetes literature looking at 
intensive lifestyle intervention including weekly counseling, meal replacements, a diet of 1,200 to $1,800 \mathrm{kcal}$ daily and moderate exercise of at least 175 minutes per week. These have resulted in an average of $8.6 \%$ weight loss at one year (25). These modest results are difficult to maintain as many in this study experienced gradual weight regain and biochemical studies have even shown the appetite stimulating hormones often revert back to pre-weight loss levels and make sustaining these changes difficult $(25,26)$. As with all studies of lifestyle modifications, there is also some element of the Hawthorne effect that should be considered as well as the resources necessary to implement these changes; this suggests that these results are likely the best outcome from lifestyle modification. These psychosocial and hormonal barriers can make it difficult for patients and providers to implement sustained changes before and after reconstruction. To go further, some expert opinion would even dictate that weight loss alone would not be effective to treat AABP due to the chronic inflammation creating permanent fibrotic skin changes that won't resolve without reconstruction (9). As highlighted previously, weight loss can be effective in limiting some complications of reconstruction but this should not be a stand alone method of management. Further guidance and knowledge of the condition can help primary care providers refer patients to the correct resources for a more lasting solution.

While referral is the ultimate goal, access to reconstructive surgeons capable of correcting AABP is not guaranteed. Buried penis reconstruction is not a universal standard of fellowships for the Society of Genito-urinary Reconstructive Surgeons (GURS) who require programs to meet minimum requirements in two of six categories: male urethral reconstruction, male incontinence surgery, male sexual health, genital reconstruction (including gender confirming surgery), urinary diversion/ureteral reconstruction or female urethral reconstruction (including female continence surgery, prolapse repair, urethral diverticulum, nerve stimulation). In terms of genital reconstruction, one study found the median number of annual cases to be 14 across accredited programs, which ranked fifth of the six categories needed to establish a fellowship (27). This data hints at the availability of surgeons experienced in the management of AABP but the true number and location of Urologic providers is unknown. In all, providers can encounter difficulty in finding guidance on the condition as well as finding a reconstructive surgeon to whom patients can be referred.

\section{Conclusions}

Literature has illustrated that AABP is a condition with significant morbidity and even the possibility of mortality for patients who it affects. Surgical management has improved drastically since initial reports and has undergone standardization in many ways to better care for patients. As we have better understood how to care for the morbidity of the condition, new questions arise in the delivery of this care to patients.

\section{Acknowledgments}

Funding: None.

\section{Footnote}

Provenance and Peer Review: This article was commissioned by the Guest Editors (Francisco E. Martins and Tobias S. Köhler) for the series "Controversies and Considerations of Penile Surgery" published in Translational Andrology and Urology. The article has undergone external peer review.

Reporting Checklist: The authors have completed the Narrative Review Checklist. Available at: http://dx.doi. org/10.21037/tau-20-1232

Peer Review File: Available at http://dx.doi.org/10.21037/ tau-20-1232

Conflicts of Interest: The authors have completed the ICMJE uniform disclosure form (available at http://dx.doi. org/10.21037/tau-20-1232). The series "Controversies and Considerations of Penile Surgery" was commissioned by the editorial office without any funding or sponsorship. The authors have no other conflicts of interest to declare.

Ethical Statement: The authors are accountable for all aspects of the work in ensuring that questions related to the accuracy or integrity of any part of the work are appropriately investigated and resolved.

Open Access Statement: This is an Open Access article distributed in accordance with the Creative Commons Attribution-NonCommercial-NoDerivs 4.0 International License (CC BY-NC-ND 4.0), which permits the noncommercial replication and distribution of the article with the strict proviso that no changes or edits are made and the 
original work is properly cited (including links to both the formal publication through the relevant DOI and the license). See: https://creativecommons.org/licenses/by-nc-nd/4.0/.

\section{References}

1. Pestana IA, Greenfield JM, Walsh M, et al. Management of "buried" penis in adulthood: an overview. Plast Reconstr Surg 2009;124:1186-95.

2. Hales CM, Carroll MD, Fryar CD, Ogden CL. Prevalence of obesity among adults and youth: United States, 20152016. National Center for Health Statistics (U.S.), editor. Available online: https://stacks.cdc.gov/view/cdc/49223

3. Rybak J, Larsen S, Yu M, et al. Single center outcomes after reconstructive surgical correction of adult acquired buried penis: measurements of erectile function, depression, and quality of life. J Sex Med 2014;11:1086-91.

4. Liaw A, Rickborn L, McClung C. Incidence of urethral stricture in patients with adult acquired buried penis. Adv Urol 2017;2017:7056173.

5. Gao B, Bo Q, Lu J, et al. Effect of surgical repair of acquired buried penis on sexual function in adults. Int Urol Nephrol 2020;52:1087-1091.

6. Fuller TW, Pekala K, Theisen KM, et al. Prevalence and surgical management of concurrent adult acquired buried penis and urethral stricture disease. World J Urol 2019;37:1409-13.

7. Theisen KM, Fuller TW, Rusilko P. Surgical management of adult-acquired buried penis: impact on urinary and sexual quality of life outcomes. Urology 2018;116:180-4.

8. Keyes EL. Urology: Diseases of the Urinary Organs, Diseases of the Male Genital Organs, the Venereal Diseases. D. Appleton; 1917.

9. Fuller TW, Theisen KM, Shah A, et al. Surgical management of adult acquired buried penis. Curr Urol Rep 2018;19:22.

10. Fuller TW, Theisen K, Rusilko P. Surgical management of adult acquired buried penis: escutcheonectomy, scrotectomy, and penile split-thickness skin graft. Urology 2017;108:237-8.

11. Jun MS, Gallegos MA, Santucci RA. Contemporary management of adult-acquired buried penis. BJU Int 2018;122:713-5.

12. Hesse MA, Israel JS, Shulzhenko NO, et al. The surgical treatment of adult acquired buried penis syndrome: a new classification system. Aesthet Surg J 2019;39:979-88.

13. Pariser JJ, Soto-Aviles OE, Miller B, et al. A simplified adult acquired buried penis repair classification system with an analysis of perioperative complications and urethral stricture disease. Urology 2018;120:248-52.

14. Monn MF, Socas J, Mellon MJ. The Use of Full Thickness
Skin Graft Phalloplasty During Adult Acquired Buried Penis Repair. Urology 2019;129:223-7.

15. Erpelding SG, Hopkins M, Dugan A, et al. Outpatient surgical management for acquired buried penis. Urology 2019;123:247-51.

16. Pekala KR, Pelzman D, Theisen KM, et al. The prevalence of penile cancer in patients with adult acquired buried penis. Urology 2019;133:229-33.

17. Barnes KT, McDowell BD, Button A, et al. Obesity is associated with increased risk of invasive penile cancer. BMC Urol 2016;16:42.

18. Hampson LA, Muncey W, Chung PH, et al. Surgical and functional outcomes following buried penis repair with limited panniculectomy and split-thickness skin graft. Urology 2017;110:234-8.

19. Voznesensky MA, Lawrence WT, Keith JN, et al. Patientreported social, psychological, and urologic outcomes after adult buried penis repair. Urology 2017;103:240-4.

20. Hughes DB, Perez E, Garcia RM, et al. Sexual and overall quality of life improvements after surgical correction of "buried penis." Ann Plast Surg 2016;76:532-5.

21. Tang SH, Kamat D, Santucci RA. Modern management of adult-acquired buried penis. Urology 2008;72:124-7.

22. Donatucci CF, Ritter EF. Management of the buried penis in adults. J Urol 1998;159:420-4.

23. Tausch TJ, Tachibana I, Siegel JA, et al. Classification system for individualized treatment of adult buried penis syndrome. Plast Reconstr Surg 2016;138:703-11.

24. Aubé M, Chua M, DeLong J, et al. Predictors of surgical complications and evaluation of outcomes after surgical correction of adult-acquired buried penis. Int Urol Nephrol 2020;52:687-92.

25. Look AHEAD Research Group; Wing RR, Bolin P, et al. Cardiovascular effects of intensive lifestyle intervention in type 2 diabetes. N Engl J Med 2013;369:145-54.

26. Sumithran P, Prendergast LA, Delbridge E, et al. Longterm persistence of hormonal adaptations to weight loss. N Engl J Med 2011;365:1597-604.

27. Saavedra AA, Rourke KF. Training in reconstructive urology: the past, present and future. Transl Androl Urol 2018;7:666-72.

Cite this article as: Staniorski CJ, Rusilko PJ. The concealed morbidity of buried penis: a narrative review of our progress in understanding adult-acquired buried penis as a surgical condition. Transl Androl Urol 2021;10(6):2536-2543. doi: $10.21037 /$ tau-20-1232 